\title{
Numerical Simulation of Jet Issuing Diagonally Upward into Density-Stratified Fluid in Cylindrical Tank
}

\author{
Tomohiro Degawa1 ${ }^{*}$, Tomomi Uchiyama1, Isao Aozasa ${ }^{2}$, Akira Ishikawa ${ }^{3}$, Koji Motoyama ${ }^{3}$ \\ ${ }^{1}$ Institute of Materials and Systems for Sustainability, Nagoya University, Nagoya, Japan \\ ${ }^{2}$ Mitsubishi Electric Co., Tokyo, Japan \\ ${ }^{3}$ Chubu Electric Power Co., Inc., Nagoya, Japan \\ Email: degawa@imass.nagoya-u.ac.jp
}

How to cite this paper: Degawa, T., Uchiyama, T., Aozasa, I., Ishikawa, A. and Motoyama, K. (2018) Numerical Simulation of Jet Issuing Diagonally Upward into Density-Stratified Fluid in Cylindrical Tank. Journal of Power and Energy Engineering, 6, 38-52.

https://doi.org/10.4236/jpee.2018.63004

Received: December 26, 2017

Accepted: March 26, 2018

Published: March 29, 2018

Copyright $\odot 2018$ by authors and Scientific Research Publishing Inc. This work is licensed under the Creative Commons Attribution International License (CC BY 4.0).

http://creativecommons.org/licenses/by/4.0/

\begin{abstract}
This study simulates the behavior of a jet issuing into a two-layer density-stratified fluid in a cylindrical tank and the resulting mixing phenomena. The upper and lower fluids are water and an aqueous solution of sodium chloride $(\mathrm{NaCl})$, respectively, with the lower fluid issuing diagonally upward from a nozzle on the bottom of the tank. The angle between the centerline of the jet and the tank bottom is $60^{\circ}$. The phenomena when the Reynolds number Re of the jet is 475,1426 , and 2614 are simulated. The mass concentration of the aqueous solution of $\mathrm{NaCl}$ is 0.02 . The simulation successfully grasps the jet behavior and the resulting mixing, which agree with the authors' experimental results at the corresponding Re value. The secondary flows that appear in the horizontal cross-sections consist of a pair of vortices and flows along the tank wall. The secondary flow at the density interface represents the intrusion of an internal density current, which gives rise to mixing along the interface.
\end{abstract}

\section{Keywords}

Jet, Density-Stratified Fluid, Mixing, Secondary Flow, Density Current

\section{Introduction}

To efficiently store liquefied natural gas (LNG), many kinds of LNG from different production areas are stored in the same LNG tank. As the density of LNG is specific to the production area, density stratification sometimes occurs when one batch of LNG is loaded on top of another batch of LNG in the same storage tank. Density-stratified LNG frequently becomes unstable over time because of 
variations in temperature and composition. When the density of the lower LNG layer becomes lower than that of the upper layer, sudden mixing, known as rollover, occurs. Rollover generates large amounts of vaporized gases, which cause severe damage to the storage tank due to over pressurization. Thus, the prevention and elimination of LNG stratification is essential for the operation of LNG storage tanks.

Mixing phenomena of density-stratified fluids induced by a jet in tanks have thus far been studied via laboratory-based experiments [1] [2] [3] [4]. One of the authors [5] performed a laboratory-based experiment to investigate a jet issuing into a two-layer density-stratified fluid in a tank and the resulting mixing phenomena. The upper and lower fluids were water and an aqueous solution of sodium chloride $(\mathrm{NaCl})$, respectively, and the lower fluid issued vertically upward from a nozzle on the bottom of the tank. The experiment confirmed that the behavior of the jet relative to the density interface is determined by the Reynolds number Re of the jet and the mass concentration of the lower fluid. It also clarified the mixing phenomena caused by the jet. The authors [6] also conducted a numerical simulation under the same conditions as those in the previous experiment [5] and confirmed the validity of the simulation method.

The authors' abovementioned experiment and simulation were performed to investigate the behavior of a jet issuing vertically upward from a nozzle on the bottom of a rectangular tank. LNG storage tanks have a cylindrical shape, and a jet frequently issues diagonally upward from a nozzle on the bottom of the tank. In a cylindrical tank, a jet colliding with the wall of the tank is considered to flow along the wall, producing circulating flow in the tank. To investigate such secondary flow and the resulting mixing phenomena via a laboratory-based experiment, the authors [7] studied the behavior of a jet issuing diagonally upward from a nozzle mounted on the bottom of a cylindrical tank. The upper and lower fluids were water and an aqueous solution of $\mathrm{NaCl}$, respectively, and the lower fluid issued from the nozzle. The angle between the jet centerline and the bottom of the tank was $60^{\circ}$. The investigation revealed secondary flows in horizontal cross-sections of the tank. It also clarified the effect of the Re value of the jet on the behavior of the jet and the resulting mixing phenomena.

This study aims to complement the authors' experimental investigation [7]. It simulates a jet issuing diagonally upward from a nozzle into a two-layer density stratified fluid in a cylindrical tank and the resulting mixing phenomena. The flows in the central vertical cross-section of the tank passing through the jet centerline and the horizontal cross-section at the density interface are favorably clarified. The simulations numerically demonstrate the effect of Reynolds number of the jet on progress of mixing.

\section{Basic Equations and Numerical Method}

\subsection{Governing Equations}

This study simulates a jet issuing into a two-layer density-stratified fluid con- 
sisting of water and an aqueous solution of $\mathrm{NaCl}$ in a tank. The simulation uses the Boussinesq approximation, which assumes that the effect of the difference in density appears only in the gravitational term. It also employs the immersed boundary (IB) method [8] to represent the wall of the tank. In this case, the mass and momentum conservation equations are expressed as:

$$
\begin{gathered}
\nabla \cdot \boldsymbol{u}=0 \\
\frac{\partial \boldsymbol{u}}{\partial t}+(\boldsymbol{u} \cdot \nabla) \boldsymbol{u}=-\frac{1}{\rho_{w}} \nabla p+v \nabla^{2} \boldsymbol{u}-\frac{\rho_{w}-\rho}{\rho_{w}} \boldsymbol{g}+\lambda \chi\left(\boldsymbol{u}_{s}-\boldsymbol{u}\right)
\end{gathered}
$$

where $\rho_{w}$ and $v$ are the density and kinematic viscosity of water, respectively, and $\boldsymbol{g}$ is the gravitational acceleration. The final term on the right-hand side of Equation (2) is a penalization term [8] for non-slip conditions on the wall of the tank, and $\lambda, \chi$, and $\boldsymbol{u}_{s}$ are the penalization parameter, mask function, and velocity of the wall, respectively.

The density $\rho$ in Equation (2) is given by the following equation:

$$
\rho=(1-C) \rho_{w}+C \rho_{s}
$$

Here, $C$ and $\rho_{s}$ are the concentration and density of $\mathrm{NaCl}$, respectively, and $C$ is governed by the following convection-diffusion equation:

$$
\frac{\partial C}{\partial t}+(\boldsymbol{u} \cdot \nabla) C=\kappa \nabla^{2} C
$$

where $\kappa$ is the diffusion coefficient of $\mathrm{NaCl}$.

\subsection{Numerical Simulation Method}

Equations (1) and (2) are solved by the simplified marker and cell (SMAC) method [9]. To solve Equation (4), the constrained interpolation profile (CIP) method [10] is employed so as to capture accurately the density interface of the density-stratified fluid.

The mask function $\chi$ on the right-hand side of Equation (2) is a step function, which is 0 within the fluid but 1 in other regions. When introducing a signed distance function $\zeta$, of which the absolute value represents the distance from the wall of the tank, $\chi$ satisfies the following relations:

$$
\chi\left(\zeta^{*}\right)= \begin{cases}1 & \zeta^{*} \leq 0 \\ 0 & \zeta^{*}>0\end{cases}
$$

where $\zeta$ is the function $\zeta$ normalized by the width of the computational grid, which takes negative and positive values on the outside of the wall and in the fluid, respectively.

Because $\chi$, as given by Equation (5), is not continuous at the wall of the tank, this study uses a revised mask function $\chi_{1}$ instead of $\chi$ to guarantee continuity:

$$
\chi_{1}\left(\zeta^{*}\right)=\left\{\begin{array}{lc}
1 & \zeta^{*}<0 \\
\left(\zeta^{*}-1\right)^{2} & 0 \leq \zeta^{*} \leq 1 \\
0 & \zeta^{*}>1
\end{array}\right.
$$


Figure 1 shows the values of $\chi$ and $\chi_{1}$, where $\chi_{1}$ changes continuously from 1 to 0 around the wall at $\zeta=0$.

\section{Computational Conditions and Experimental Visualization}

\subsection{Computational Condition}

At the initial time $t=0$, a two-layer density-stratified fluid is in a static condition in a cylindrical tank, as shown in Figure 2. The upper and lower fluids are water and an aqueous solution of $\mathrm{NaCl}$, respectively. The mass concentration $C_{0}$ of the aqueous solution of $\mathrm{NaCl}$ is 0.02 . A nozzle with a diameter of $d$ is mounted on the bottom of the tank. The diameter of the tank is $30 \mathrm{~d}$. The origin of the coordinates is set at the center of the tank bottom. The $x-y$ plane is horizontal, and the $z$-axis is considered to be vertical. The nozzle is positioned at $(-10.5 d, 0,0)$, and the angle between the jet centerline and the bottom of the tank is $60^{\circ}$. An aqueous solution of $\mathrm{NaCl}$ with a concentration $C_{0}$ issues from the nozzle. A fluid outlet hole

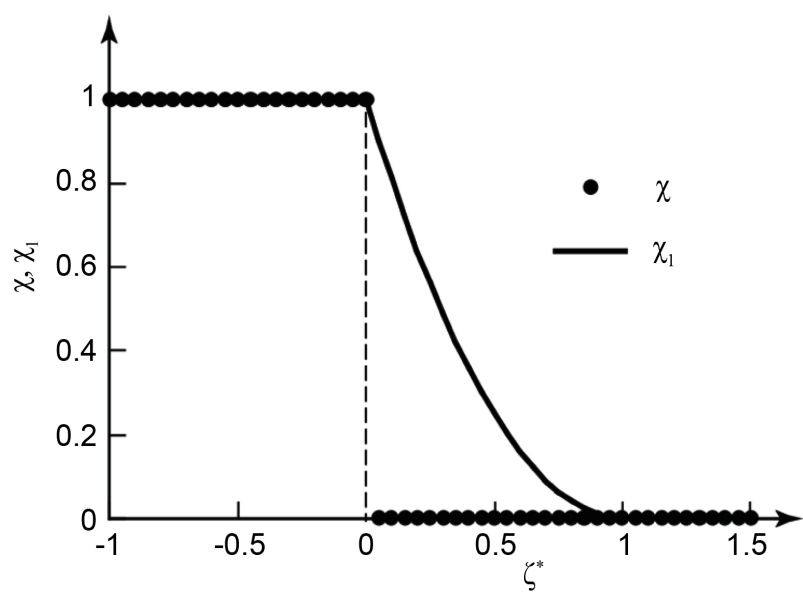

Figure 1. Mask function between the wall of the tank and the fluid.

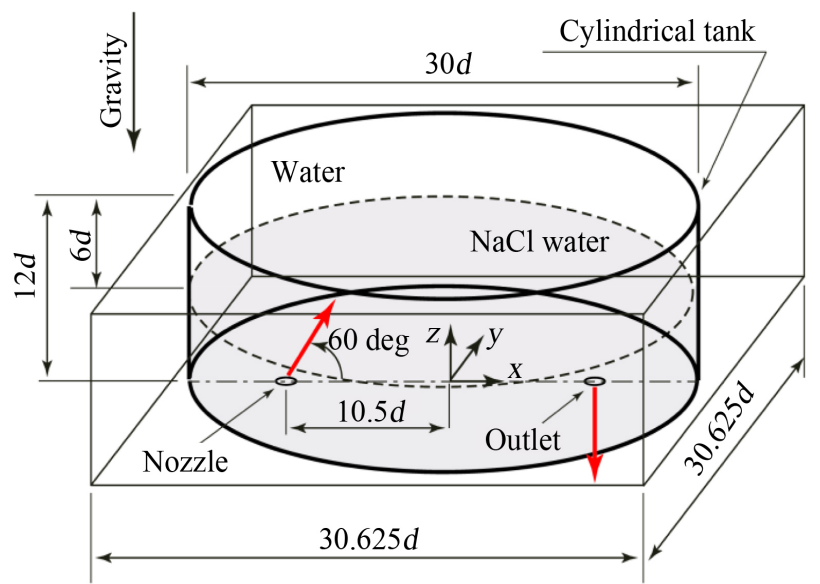

Figure 2. Two-layer density-stratified fluid in cylindrical tank and computational domain. 
with a diameter of $d$ is located at $(10.5 d, 0,0)$. The thicknesses of the upper and lower fluids are $6 d$.

Table 1 lists the computational conditions. A rectangular region $(30.625 d \times$ $30.625 d \times 12 d)$ surrounding the cylindrical tank is chosen as the computational domain. It is divided into $245 \times 245 \times 96$ grid cells. The wall of the tank is represented by the IB method using Equation (6). Non-slip conditions are imposed on the tank bottom and side wall, whereas slip conditions are applied at the upper boundary. At the nozzle exit, a parabolic distribution is assigned to the velocity. The value of Re is defined by $d U_{0} / v$, where $U_{0}$ is the mean velocity at the nozzle outlet and $v$ is the kinematic viscosity of water. The time increment $\Delta t$ is $0.0125 d / U_{0}$, and the penalization parameter $\lambda$ is set at $1 / \Delta t$.

\subsection{Computational Condition}

The authors [7] conducted an experimental study of the behavior of the jet and the resulting mixing phenomena under the same conditions as those in the present simulation. They revealed that the jet behavior relative to the density interface can be classified into three patterns (Pattern A, Pattern B, and Pattern C) according to the value of Re. The patterns for different Re values are listed in Table 2.

Pattern A: The jet reaches the interface without penetrating it but spreads almost horizontally outward along the interface.

Pattern B: The jet penetrates the interface but does not reach the upper water surface. The top of the jet falls back to the interface and spreads horizontally without penetrating the interface again.

Pattern C: The jet reaches the upper water surface, spreads along the surface, and falls back to the interface.

The authors' experimental study [7] captured images of the flow in the central vertical cross-section $(y=0)$ passing through the jet centerline. Figure 3 shows images for $\operatorname{Re}=475,1426$, and 2614, which represent Pattern A, Pattern

Table 1. Simulation conditions.

$\begin{array}{cc}\text { Nozzle diameter } & 10 \mathrm{~mm} \\ \text { Angle of nozzle to horizontal }(x) \text { axis } & 60^{\circ} \\ \text { Tank diameter } & 30 d \\ \text { Upper fluid } & \text { Water } \\ \text { Lower fluid } & \text { Aqueous solution of } \mathrm{NaCl} \\ \text { Thickness of upper fluid } & 6 d \\ \text { Thickness of lower fluid } & 6 d \\ \text { Initial concentration of aqueous solution of } \mathrm{NaCl} C_{0} & 0.02 \\ \text { Reynolds number Re }=d U_{0} / v & 475,1426,2614 \\ \text { Computational domain } & 30.625 d \times 30.625 d \times 12 d \\ \text { Computational grid } & 245 \times 245 \times 96 \\ \text { Time increment } \Delta t U_{0} / d & 0.0125\end{array}$


Table 2. Relationship between flow pattern and Re.

\begin{tabular}{cccccccccc}
\hline Re & 475 & 713 & 951 & 1188 & 1426 & 1663 & 1901 & 2139 & 2376 \\
\hline Pattern & A & A & B & B & B & B & B & B & B \\
\hline Re & 2614 & 2852 & 3089 & 3565 & 4040 & 4753 & & & \\
\hline Pattern & C & C & C & C & C & C & & & \\
\hline
\end{tabular}

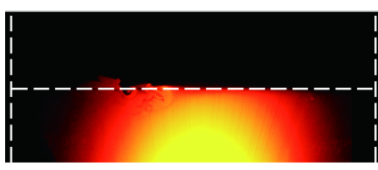

$\operatorname{Re}=475$

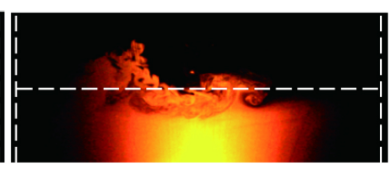

$\operatorname{Re}=1426$

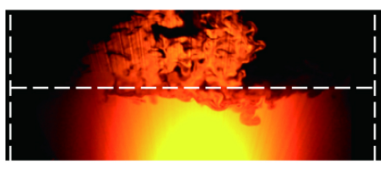

$\mathrm{Re}=2614$

Figure 3. Experimentally visualized flow patterns at $\mathrm{Re}=475,1426$, and 2614 .

B, and Pattern C, respectively. At the initial time $t=0$, a small amount of fluorescent dye (rhodamine B) was uniformly added to the lower fluid. Images in the vertical cross-section were captured by a video camera using a laser light sheet (power: $1 \mathrm{~W}$, wavelength: $532 \mathrm{~nm}$, thickness: $2 \mathrm{~mm}$ ). The concentration $C$ of $\mathrm{NaCl}$ was indicated by the brightness of the fluorescent dye. At $\operatorname{Re}=475$, local deformation of the density interface caused by the jet reaching the interface was visualized. At $\operatorname{Re}=1426$, the decline of the jet penetrating the density interface was visualized. The jet deformed the interface and gave rise to mixing along the interface. At $\mathrm{Re}=2614$, spreading of the jet along the upper surface and active mixing between the jet and the ambient fluid were visualized.

This study simulates the flow patterns at $\operatorname{Re}=475,1426$, and 2614 . The results are compared with the experimentally observed results to validate the simulation method, and the behavior of the jet and the resulting mixing phenomena are investigated closely.

\section{Results and Discussion}

\subsection{Behavior of Jet Relative to Density Interface and Its Classification}

The jet velocity, which is expressed in non-dimensional form in terms of the mean velocity $U_{0}$ at the nozzle outlet, is distributed as shown in Figures 4-6.

Figure 4 shows the distributions at $\mathrm{Re}=475$, where the results at three time points $t^{*}\left(=t U_{0} / d\right)$ of 200,400 and 600 are indicated. The distributions in the central vertical cross-section $(y=0)$ passing through the jet centerline are shown in the upper part of Figure 4. The jet, on reaching the density interface, falls back slightly without penetrating the interface and diffuses toward the tank wall facing the nozzle. Pattern A, which was experimentally visualized at $\operatorname{Re}=475$, is successfully simulated. In the horizontal cross-section $(z / d=12)$ corresponding to the density interface at the initial time $(t=0)$, the velocity reaches its maximum value where the jet reaches the interface, as 
$0\left(u_{x}^{2}+u_{z}^{2}\right)^{1 / 2} / U_{0} \quad 2$

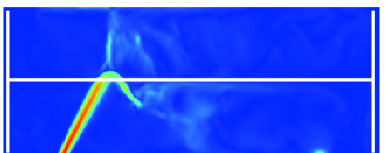

$t^{*}=200$

$0\left(u_{x}^{2}+u_{y}^{2}\right)^{1 / 2} / U_{0} 0.5$

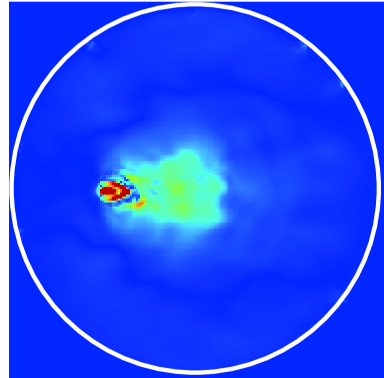

$t^{*}=200$

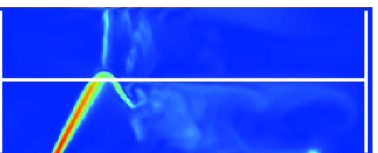

$t^{*}=400$

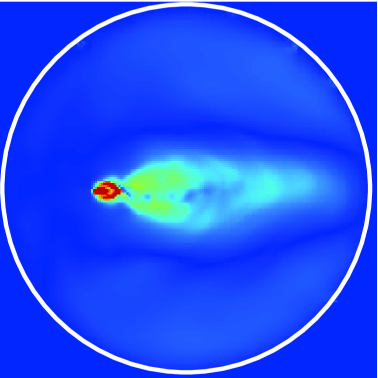

$t^{*}=400$

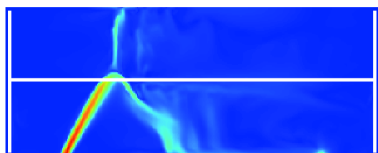

$t^{*}=600$

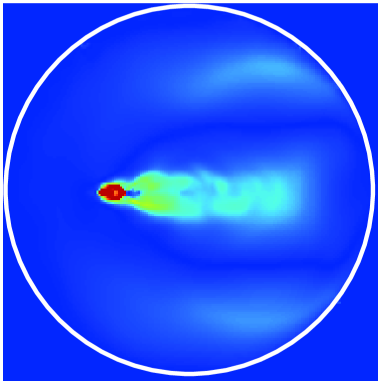

$t^{*}=600$

Figure 4. Velocity distribution at $\mathrm{Re}=475$.

$0\left(u_{x}^{2}+u_{z}^{2}\right)^{1 / 2} / U_{0} \quad 2$

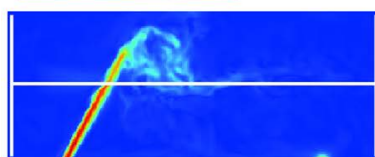

$t^{*}=200$

$0^{\left(u_{x}^{2}+u_{y}^{2}\right)^{1 / 2} / U_{0}} 0.5$

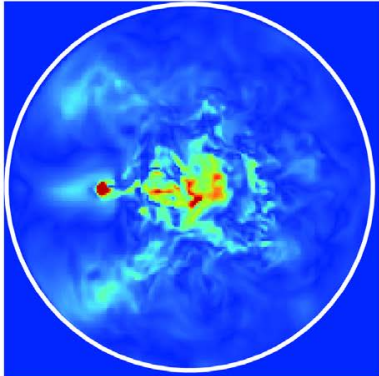

$t^{*}=200$

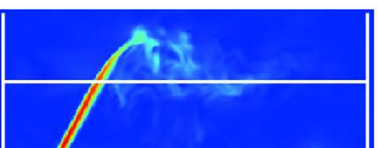

$t^{*}=400$

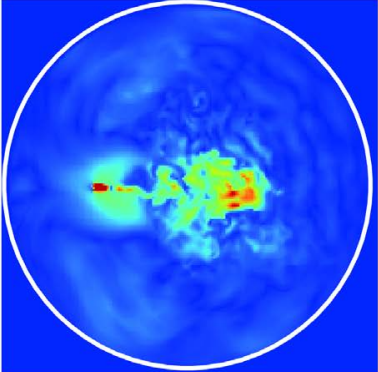

$t^{*}=400$

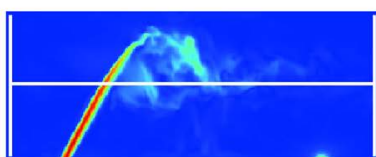

$t^{*}=600$

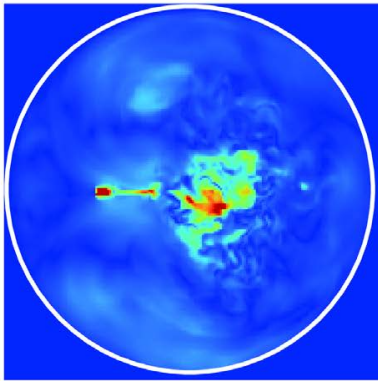

$t^{*}=600$

Figure 5. Velocity distribution at $\operatorname{Re}=1426$.

shown in the lower part of Figure 4. Horizontal flow toward the tank wall is observed, with a narrow width. It should be noted that flows along the tank wall are also simulated at $t^{\star}=600$.

Figure 5 shows the velocity distributions at $\mathrm{Re}=1426$. The jet penetrates the density interface, as is found from the distributions in the central vertical cross-section, but does not reach the upper boundary. This is because the density of the jet is larger than that of the upper fluid, and accordingly the jet falls back to the interface owing to the gravitational effect. The jet, on reaching the interface again, tends to spread horizontally along the interface. This 


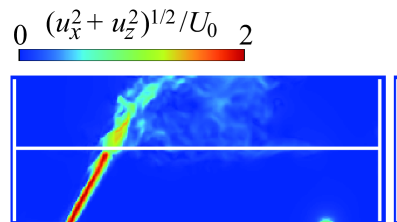

$t^{*}=200$
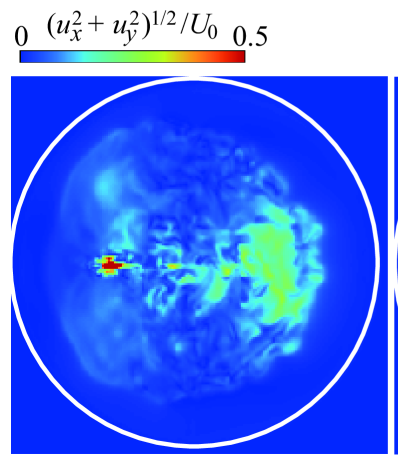

$t^{*}=200$

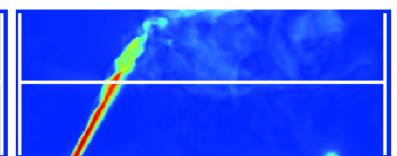

$t^{*}=400$

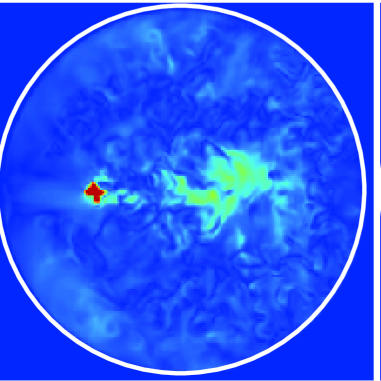

$t^{*}=400$

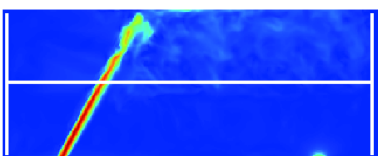

$t^{*}=600$

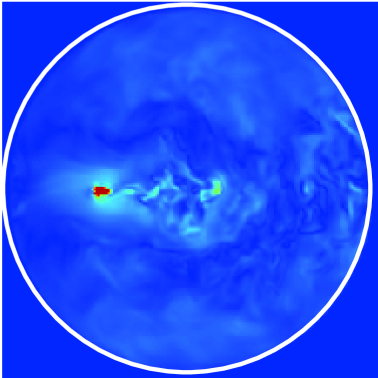

$t^{*}=600$

Figure 6. Velocity distribution at $\mathrm{Re}=2614$.

coincides with Pattern B, which was observed by the experimental visualization at $\mathrm{Re}=1426$. In the horizontal cross-section, the velocity reaches its maximum value at several locations along the $x$-axis. Horizontal flow at the density interface is reconfirmed, which originates from the downward flow of the jet after penetrating the interface.

Figure 6 shows the velocity distributions at $\operatorname{Re}=2614$. The jet reaches the upper boundary and then spreads along the boundary. In the upper fluid, the flow falling back to the density interface, as well as the horizontal flow along the interface, is simulated. Pattern $\mathrm{C}$, which was experimentally visualized at $\operatorname{Re}=2614$, is observed. When compared with the distributions at $\mathrm{Re}=1426$, the absolute value of the non-dimensional velocity at the density interface (horizontal cross-section) is low. This is because the jet penetrates the interface, and accordingly a notable effect of downward flow does not occur at the interface.

Figure 7 shows the velocity distributions in the horizontal cross-section $(z / d=6)$ at $t^{\star}=600$. This cross-section corresponds to the density interface at $t^{*}=0$. The velocity vectors, which are simulated at the grid points, are thinned so that their distribution is easily seen. At $\mathrm{Re}=475$, a pair of vortices is induced by the jet near the tank wall. At $\operatorname{Re}=1426$, a pair of vortices is generated just behind the location where the jet penetrates. At $\operatorname{Re}=2614$, the flow along the tank wall is simulated. These results suggest that secondary flows occur in this horizontal cross-section. When $\mathrm{Re}=475$, such a secondary flow was visualized in the authors' experiment [7].

Figure 8 shows the second invariant of the velocity gradient tensor $Q$ presented as the iso-surface of $Q /\left(U_{0} / d\right)^{2}=0.1$. When $\mathrm{Re}=475$ and 1426, vortical structures near the density interface are confirmed. These correspond to the 


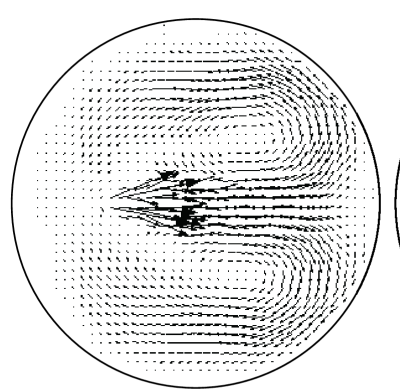

$\operatorname{Re}=475$

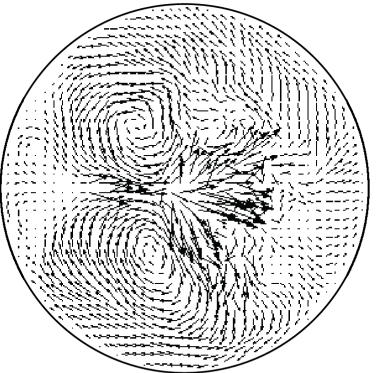

$\operatorname{Re}=1426$

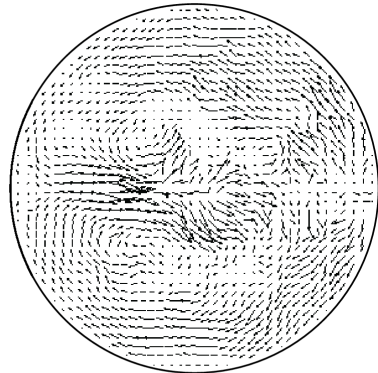

$\operatorname{Re}=2614$

Figure 7. Velocity vectors at the density interface.

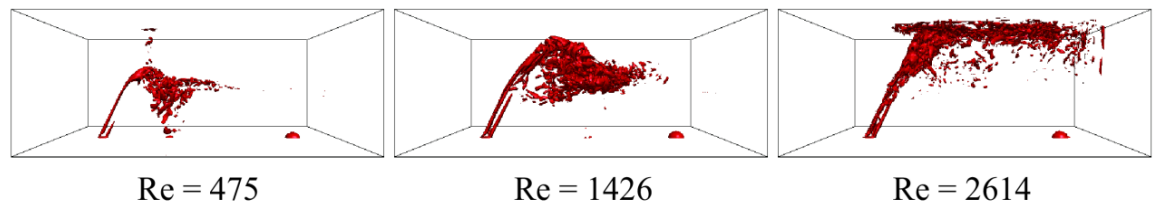

Figure 8. Iso-surface of $Q\left(Q /\left(U_{0} / d\right)^{2}=0.1\right)$.

secondary flows indicated in Figure 7.

When a fluid with a density of $\rho$ reaches a density interface from the outside, it flows in a horizontal direction along the interface if the condition $\rho_{1}<\rho<\rho_{2}$ is satisfied, where $\rho_{1}$ and $\rho_{2}$ are the densities of the upper and lower fluids, respectively [11]. Such flow is recognized as the intrusion of an internal density current. An example of this type of flow can be observed in the temperature-stratified water of a dam reservoir when muddy water mixed with sand flows into the reservoir and reaches the density interface. The secondary flow along the density interface simulated in this study corresponds to the intrusion of an internal density current.

\subsection{Concentration Distribution and Comparison with Experimental Results}

The distributions of the concentration $C$ for Pattern A, Pattern B, and Pattern C are shown in Figures 9-11, respectively. The results for the central vertical cross-section passing through the jet centerline $(y=0)$ and those for the density interface $(z / d=6)$ are depicted.

Figure 9 shows the distributions at $\mathrm{Re}=475$ for Pattern $\mathrm{A}$. The jet reaches the density interface without penetrating it. In the central vertical cross-section, the jet pushes the interface up locally. To the right of this location, the concentration decreases slightly because the downward flow entrains the upper fluid. Mixing is hardly induced by the jet. At the density interface, the concentration reaches its maximum value at the locations where the jet reaches the interface. The change in concentration within the interface is not remarkable.

Figure 10 shows the results at $\mathrm{Re}=1426$ for Pattern $\mathrm{B}$. The jet penetrates the interface but does not reach the upper boundary. Penetration through the interface is confirmed by the concentration distribution, and the concentration 


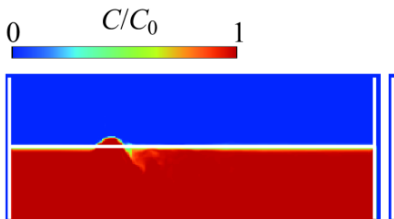

$t^{*}=200$
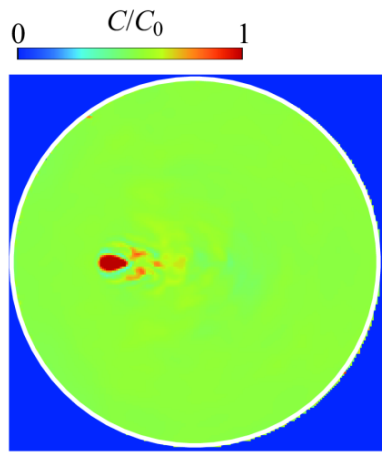

$t^{*}=200$

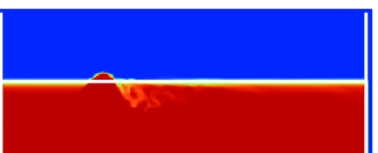

$t^{*}=400$

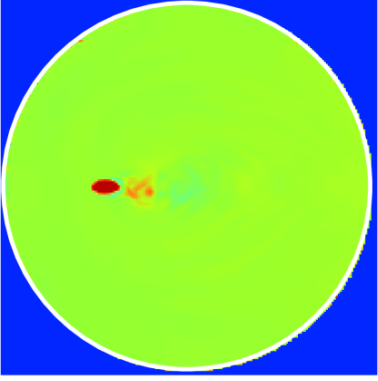

$t^{*}=400$

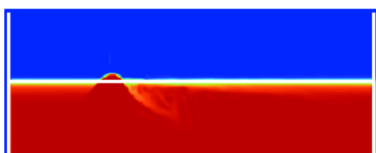

$t^{*}=600$

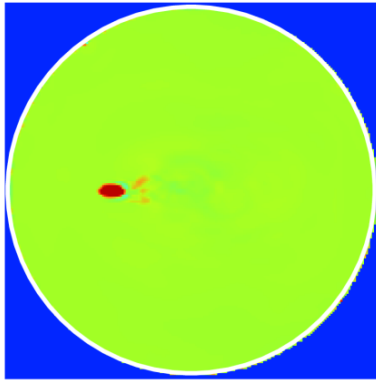

$t^{*}=600$

Figure 9. Concentration distribution at $\mathrm{Re}=475$.

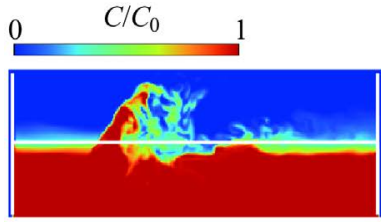

$t^{*}=200$
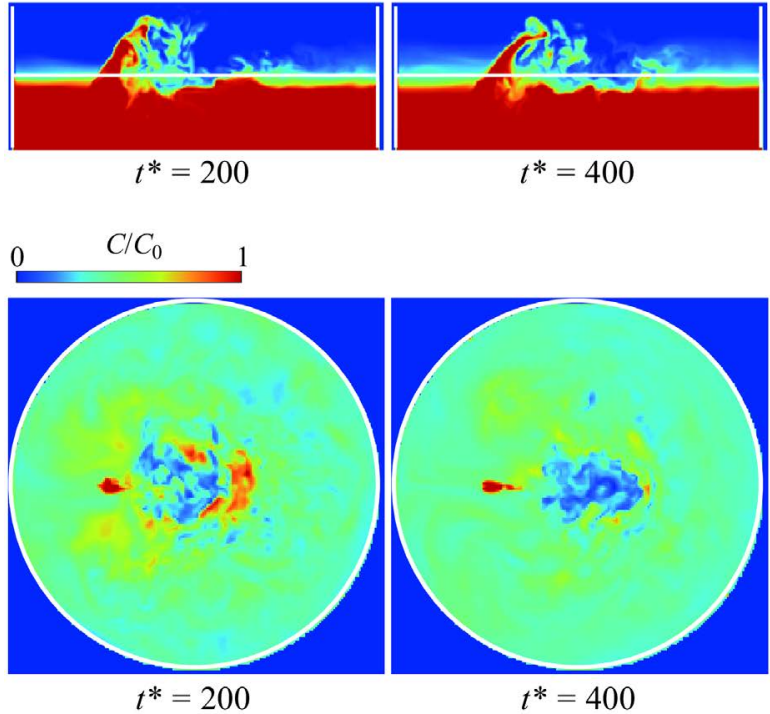

$t^{*}=400$

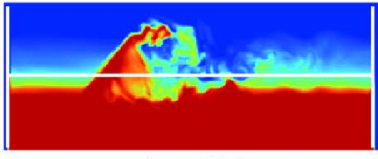

$t^{*}=600$

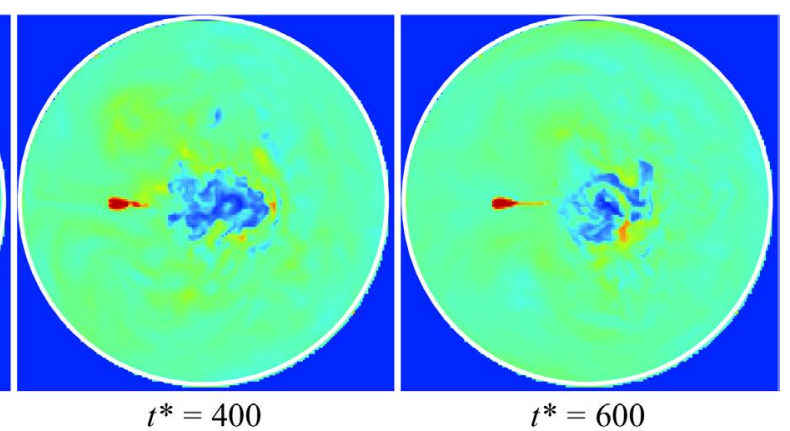

Figure 10. Concentration distribution at $\mathrm{Re}=1426$.

increases locally in the upper fluid. The downward flow deforms the interface. The deformation becomes greater with the horizontal spread of the jet. At the density interface, the concentration is unevenly distributed, and mixing proceeds in a layer region along the interface. These phenomena are caused by the secondary flow shown in Figure 7.

The concentration distributions at $\mathrm{Re}=2614$ for Pattern $\mathrm{C}$ are shown in Figure 11. The jet reaches the upper boundary. Active mixing between the jet and the ambient fluid occurs in the upper fluid. At the density interface, mixing is also generated. The concentration is relatively high and unevenly distributed. 


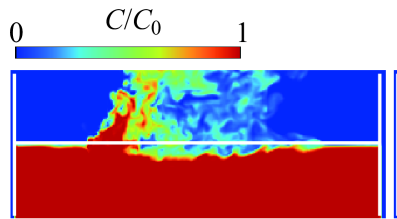

$t^{*}=200$

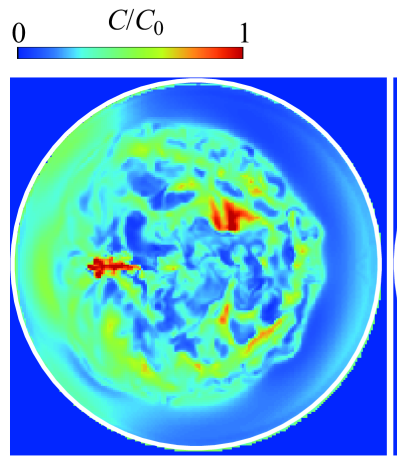

$t^{*}=200$

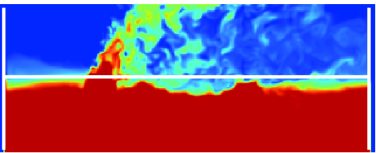

$t^{*}=400$

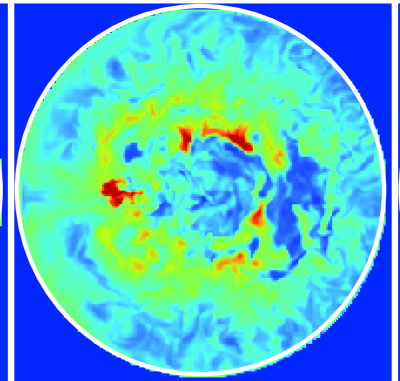

$t^{*}=400$

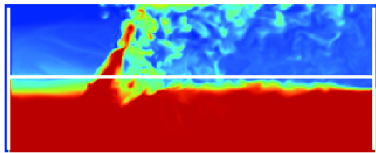

$t^{*}=600$

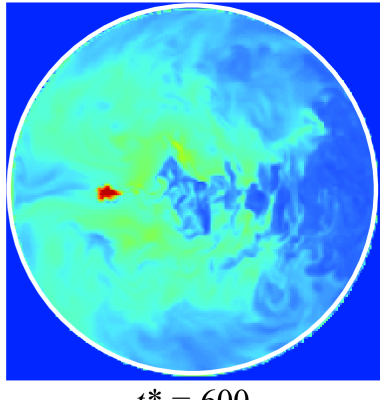

Figure 11. Concentration distribution at $\operatorname{Re}=2614$.

These phenomena are due to the secondary flow.

The simulated concentration distributions in the central vertical cross-section shown in Figures 9-11 agree with the experimentally visualized distributions [7] shown in Figure 3, which demonstrate the validity of the simulation method.

Figure 12 and Figure 13 show the velocity and concentration distributions in a horizontal cross-section at an intermediate height in the upper layer $(z / d=$ 8.4). The results at $t^{*}=600$ for $\operatorname{Re}=1426$ and 2614 are indicated. Secondary flows occur, but they are not so clear as at the density interface. It can be confirmed that the secondary flows generate the mixing phenomenon and the uneven distribution of concentration.

\subsection{Effect of Re Value on Progress of Mixing}

To estimate the progress of mixing in the tank, the root-mean-square of the concentration $C$, namely $C_{\mathrm{rms}}$, is calculated for the central vertical cross-section by the following equation:

$$
C_{\mathrm{rms}}=\left[\frac{1}{N} \sum_{i=1}^{N}\left(C_{i}-\bar{C}\right)^{2}\right]^{1 / 2}
$$

where $C_{i}$ is the value of $C$ at computational grid point $i, N$ is the number of grid points, and $\bar{C}$ is the mean value of $C$ in the cross-section. The value of $C_{\text {rms }}$ decreases during the course of mixing.

Figure 14 shows the temporal evolution of $C_{\mathrm{rms}}$ in the central vertical cross-section passing through the jet centerline $(y=0)$. When $\operatorname{Re}=475$ (Pattern A), $C_{\mathrm{rms}}$ decreases gradually with the lapse of time. This is because the jet reaches the density interface without penetrating the interface, and accordingly gradual mixing occurs locally around the interface. When $\mathrm{Re}=1426$ (Pattern B), $C_{\mathrm{rms}}$ declines markedly at $10 \leq t^{\star} \leq 84$, which is because active mixing between the jet 


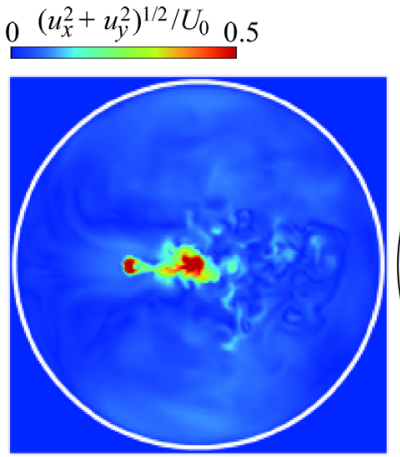

Velocity

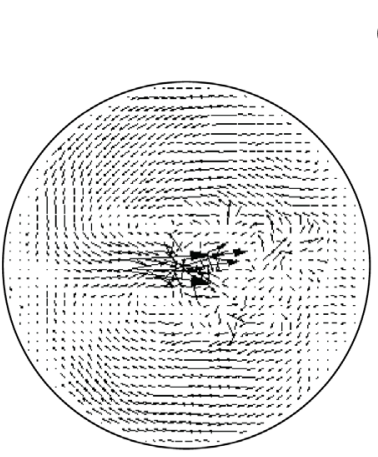

Velocity
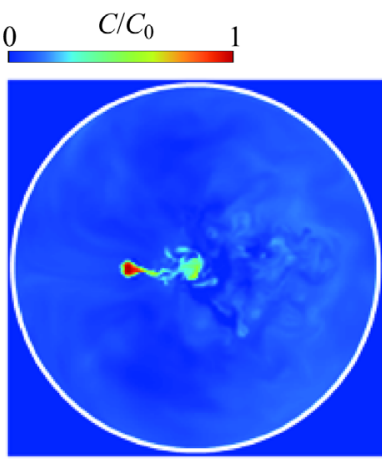

Concentration

Figure 12. Velocity and concentration distributions in horizontal cross-section $(z / d=$ 8.4) at $\mathrm{Re}=1426$.

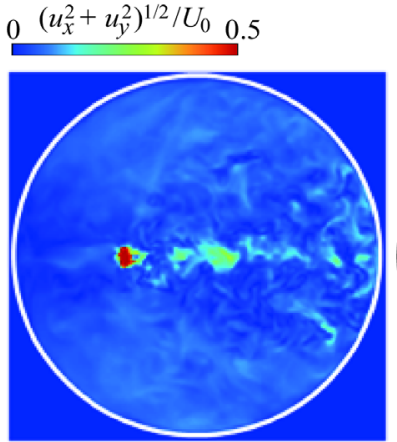

Velocity

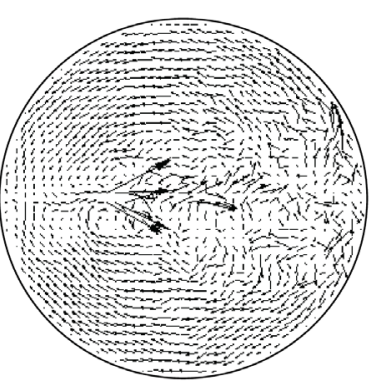

Velocity

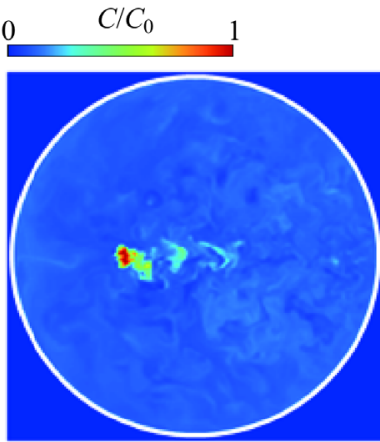

Concentration

Figure 13. Velocity and concentration distributions in horizontal cross-section $(z / d=$ 8.4) at $\mathrm{Re}=2614$.

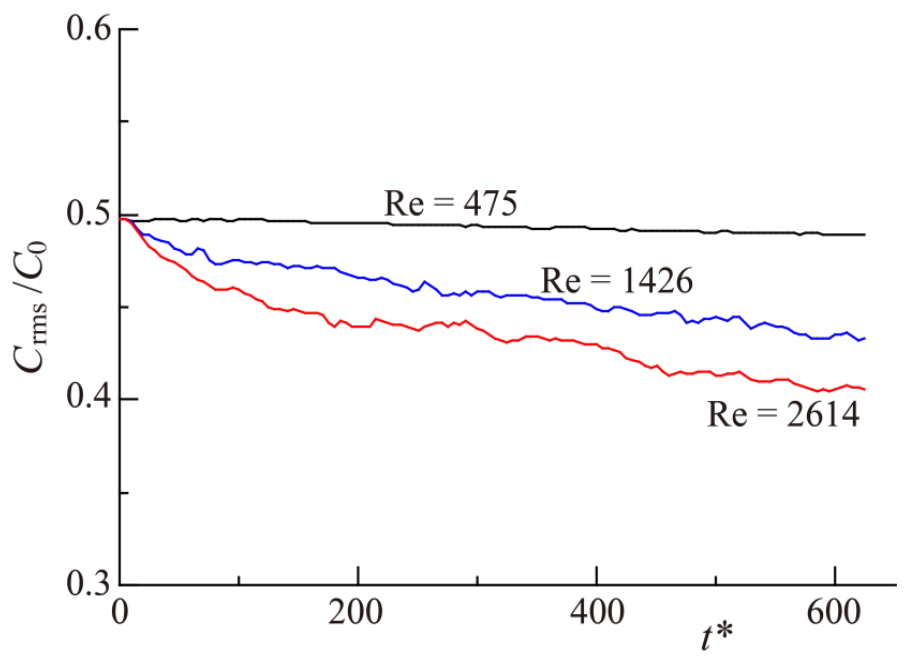

Figure 14. Temporal evolution of root-mean-square concentration in central horizontal cross-section at $y=0$.

penetrating the density interface and the ambient fluid occurs. However, the rate of change with time of $C_{\mathrm{rms}}$ becomes less at $t^{\star}>84$. This is attributable to the fact that mixing occurs only around the interface. When $\mathrm{Re}=2614$ (Pattern C), $C_{\mathrm{rms}}$ 
decreases greatly at $10 \leq t^{*} \leq 100$. During this time period, the top of the jet flows toward the upper boundary. However, the decrease is less after this period, and the rate of change with time is almost the same as that at $\operatorname{Re}=1426$. These data indicate that the rate of progress of mixing is independent of the Re value after a certain period of time in the cases of Pattern B and Pattern C.

Figure 15 shows the change in the value of $C_{\mathrm{rms}}$ in the central vertical cross-section $(x=0)$ perpendicular to the cross-section $(y=0)$ shown in Figure 14. The effect of the Re value on the decrease in $C_{\mathrm{rms}}$ is similar to that shown in Figure 14.

Figure 16 shows the temporal evolution of the mean concentration $\bar{C}$ in a horizontal cross-section at an intermediate height in the upper fluid $(z / d=8.4)$. When $\operatorname{Re}=475$ (Pattern A), the value of $\bar{C}$ remains zero. When $\operatorname{Re}=1426$ (Pattern B), the increase in $\bar{C}$ is greater at $t^{*} \geq 260$. When $\operatorname{Re}=2614$ (Pattern

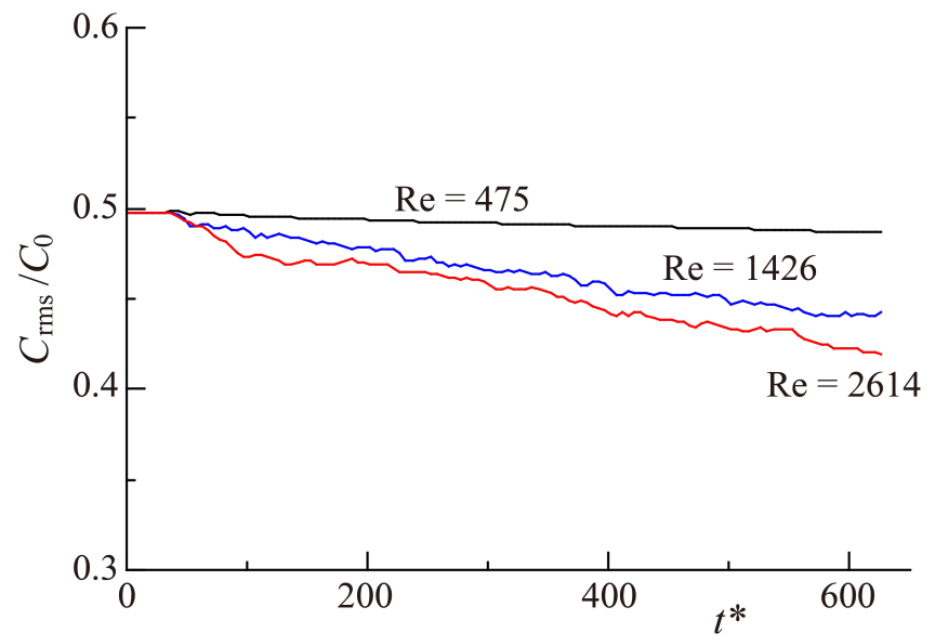

Figure 15. Temporal evolution of root-mean-square concentration in central horizontal cross-section at $x=0$.

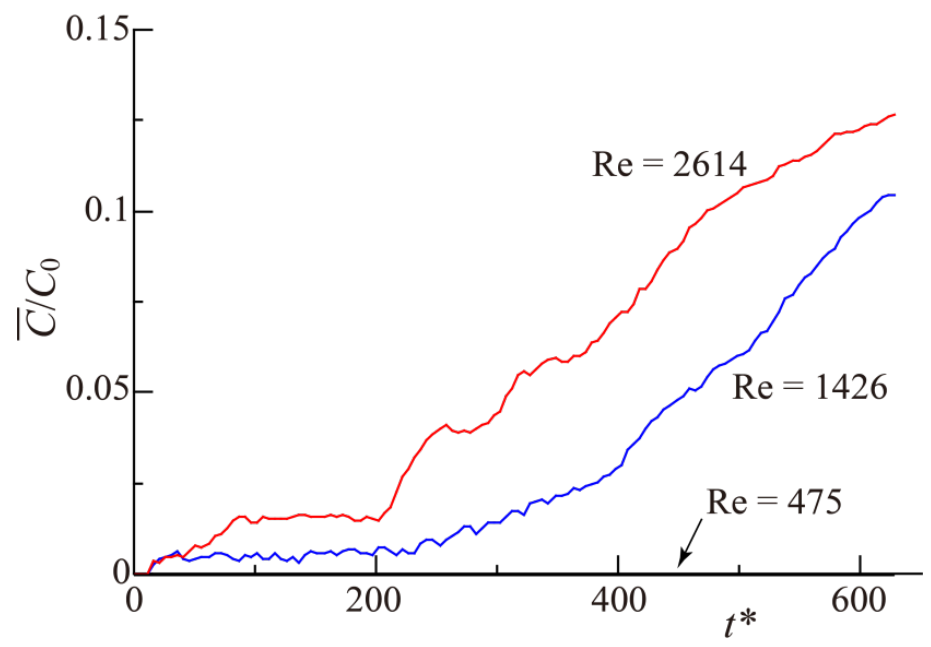

Figure 16. Temporal evolution of average concentration in horizontal cross-section at $z / d=8.4$. 
C), the increase is almost the same as that at $\operatorname{Re}=1426$, although the value of $\bar{C}$ is higher. In the cases of Pattern B and Pattern $\mathrm{C}$, the change in mean concentration in the horizontal cross-section in the upper fluid is independent of the value of Re after a certain period of time, which is similar to the result for $C_{\mathrm{rms}}$ shown in Figure 14 and Figure 15.

\section{Conclusions}

The behavior of a jet issuing into a two-layer density-stratified fluid in a cylindrical tank and the resulting mixing phenomena are numerically simulated. The upper and lower fluids are water and an aqueous solution of $\mathrm{NaCl}$, respectively, with the lower fluid issuing diagonally upward from a nozzle on the bottom of the tank. The nozzle diameter $d$ is $10 \mathrm{~mm}$, and the angle between the jet centerline and the tank bottom is $60^{\circ}$. The tank diameter is $30 d$, and the thicknesses of the upper and lower fluids are $6 d$. The phenomena for the jet Reynolds number $\operatorname{Re}$ at 475,1426 , and 2614 are simulated. The mass concentration of the aqueous solution of $\mathrm{NaCl}$ is 0.02 . The results are summarized as follows:

1) The behavior of the jet relative to the density interface can be classified into three patterns (Pattern A, Pattern B, and Pattern C) by the value of Re, in the same manner as in the corresponding experiment. In Pattern $\mathrm{A}$ at $\operatorname{Re}=475$, the jet reaches the interface without penetrating it but spreads almost horizontally outward along the interface. In Pattern B at $\mathrm{Re}=1426$, the jet penetrates the interface but does not reach the upper boundary. The top of the jet falls back to the interface and spreads horizontally without penetrating the interface again. In Pattern $\mathrm{C}$ at $\mathrm{Re}=2614$, the jet reaches the upper boundary, spreads along the surface, and falls back to the interface.

2) In Pattern A, mixing progresses locally near the density interface. In Pattern $B$, mixing occurs in a layer region along the interface. In Pattern $C$, active mixing appears in the entire region of the upper and lower fluids. These phenomena agree with the experimentally visualized results.

3) A flow in a horizontal direction occurs at the density interface. This corresponds to the intrusion of an internal density current. The flow consists of secondary flow such as a pair of vortices and flows along the tank wall. This flow causes a change in concentration distribution at the density interface.

4) In Pattern B and Pattern C, the rate of progress of mixing is independent of the value of Re after a certain period of time.

\section{References}

[1] Baines, W.D., Turner, J.S. and Campbell, I.H. (1990) Turbulent Fountains in an Open Chamber. Journal of Fluid Mechanics, 212, 557-592. https://doi.org/10.1017/S0022112090002099

[2] Bloomfield, L.J. and Kerr, R.C. (1998) Turbulent Fountains in a Stratified Fluid. Journal of Fluid Mechanics, 358, 335-356. https://doi.org/10.1017/S0022112097008252

[3] Lin, Y.J.P. and Linden, P.F. (2005) The Entrainment Due to a Turbulent Fountain at 
a Density Interface. Journal of Fluid Mechanics, 542, 25-52. https://doi.org/10.1017/S002211200500635X

[4] Ansong, J.K., Kyba, P.J. and Sutherland, B.R. (2008) Fountains Impinging upon a Density Interface. Journal of Fluid Mechanics, 595, 115-139. https://doi.org/10.1017/S0022112007009093

[5] Shakouchi, S., Fukue, S. and Uchiyama, T. (2015) Investigation of the Behavior of a Jet Issued into Two-Layer Density-Stratified Fluid. Journal of Flow Control, Measurement and Visualization, 3, 1-9. https://doi.org/10.4236/jfcmv.2015.31001

[6] Shakouchi, S., Shimada, S. and Uchiyama, T. (2015) Numerical Simulation of Jet Flow Issued into Density-Stratified Fluid by Vortex in Cell Method. Advances and Applications in Fluid Mechanics, 17, 115-134. https://doi.org/10.17654/15-AAFM-01701-115

[7] Degawa, T., Fukue, S. and Uchiyama, T. (2017) Behavior of a Jet Issued Diagonally Upward into Two-Layer Density-Stratified Fluid in a Cylindrical Tank. Journal of Flow Control, Measurement and Visualization, 5, 51-64. https://doi.org/10.4236/jfcmv.2017.53004

[8] Angot, P., Bruneau, C.-H. and Fabrie, P. (1999) A Penalization Method to Take into Account Obstacles in Incompressible Viscous Flows. Numerische Mathematik, 81, 497-520. https://doi.org/10.1007/s002110050401

[9] Amsden, A.A. and Harlow, F.H. (1970) The SMAC Method: A Numerical Technique for Calculating Incompressible Fluid Flows. Los Alamos Scientific Laboratory Report LA-4370.

[10] Takewaki, H., Nishiguchi, A. and Yabe, T. (1985) Cubic Interpolated Pseudo-Particle Method (CIP) for Solving Hyperbolic-Type Equations. Journal of Computational Physics, 61, 261-268. https://doi.org/10.1016/0021-9991(85)90085-3

[11] Simpson, J.E. (1982) Gravity Currents in the Laboratory, Atmosphere, and Ocean. Annual Review of Fluid Mechanics, 14, 213-234.

https://doi.org/10.1146/annurev.fl.14.010182.001241 